\title{
PARRONDO'S GAME MODEL TO FIND NUMERICALLY THE STABLE ATTRACTORS OF A TUMOR GROWTH MODEL
}

\author{
MARIUS-F. DANCA \\ Department of Mathematics and Computer Science, Avram Iancu University, \\ Str. Ilie Macelaru, nr. 1A, 400380 Cluj-Napoca, Romania \\ and \\ Romanian Institute of Science and Technology, \\ Str. Ciresilor nr. 29, 400487 Cluj-Napoca, Romania \\ DEJIAN LAI \\ Division of Biostatistics, University of Texas, School of Public Health, \\ RAS W1006, Houston, TX 77030, USA \\ and \\ Faculty of Statistics, Jiangxi University of Finance and Economics \\ Nanchang, Jiangxi, China
}

\begin{abstract}
In this letter we present a simple and accessible way to enhance the stable behaviors of a chaotic dynamical system which models a cancer growth, presented in [Itik \& Banks, 2010]. The algorithm presented in [Danca et al., 2011], approximates numerically any attractor of a system belonging to a defined class of dynamical systems, by alternating the control parameter in relatively short periods of time. Switching the control parameter within a set of values corresponding to some chaotic behaviors, the result may be a stable evolution or, reversely a chaotic behavior may be obtained by switching the parameter within a set of values corresponding to stable evolutions. This apparently surprising phenomenon is in fact a generalization of the known Parrondo's paradox.
\end{abstract}

\section{Introduction}

There are several mathematical models describing cancer behavior (see e.g. [Gatenby et al., 2003] or [Roose et al., 2007]). These were generally divided into models described by diffusion partial differential equations, and by ordinary differential equations. Since tumor diseases are one of the greatest killers, their stabilization is biologically motivated. In this letter we use a simple and accessible way to enhance any stable attractors of a chaotic dynamical system presented in [Itik \& Banks, 2010], which models a cancer growth being described by the following set of three-dimensional ODEs

$$
\begin{aligned}
& \dot{x}_{1}=x_{1}\left(1-x_{1}\right)-x_{1} x_{2}-2.5 x_{1} x_{3}, \\
& \dot{x}_{2}=0.6 x_{2}\left(1-x_{2}\right)-1.5 x_{1} x_{2}, \\
& \dot{x}_{3}=\frac{4.5 x_{1} x_{3}}{x_{1}+1}-0.2 x_{1} x_{3}-p x_{3},
\end{aligned}
$$

with $p$ the positive real control parameter. Its reach dynamics are revealed by the reverse bifurcations scenarios (see Figures 1, 2 for the component $x_{2}$ ). To obtain the stable behaviors, we need a set of parameter values $P_{N}=\left\{p_{1}, p_{2}, \ldots, p_{N}\right\}$ and a single-step scheme for ODEs (here the standard RK method) with step-size $h$. The utilized algorithm, named hereafter Parameter Switching (PS), allows the numerical 


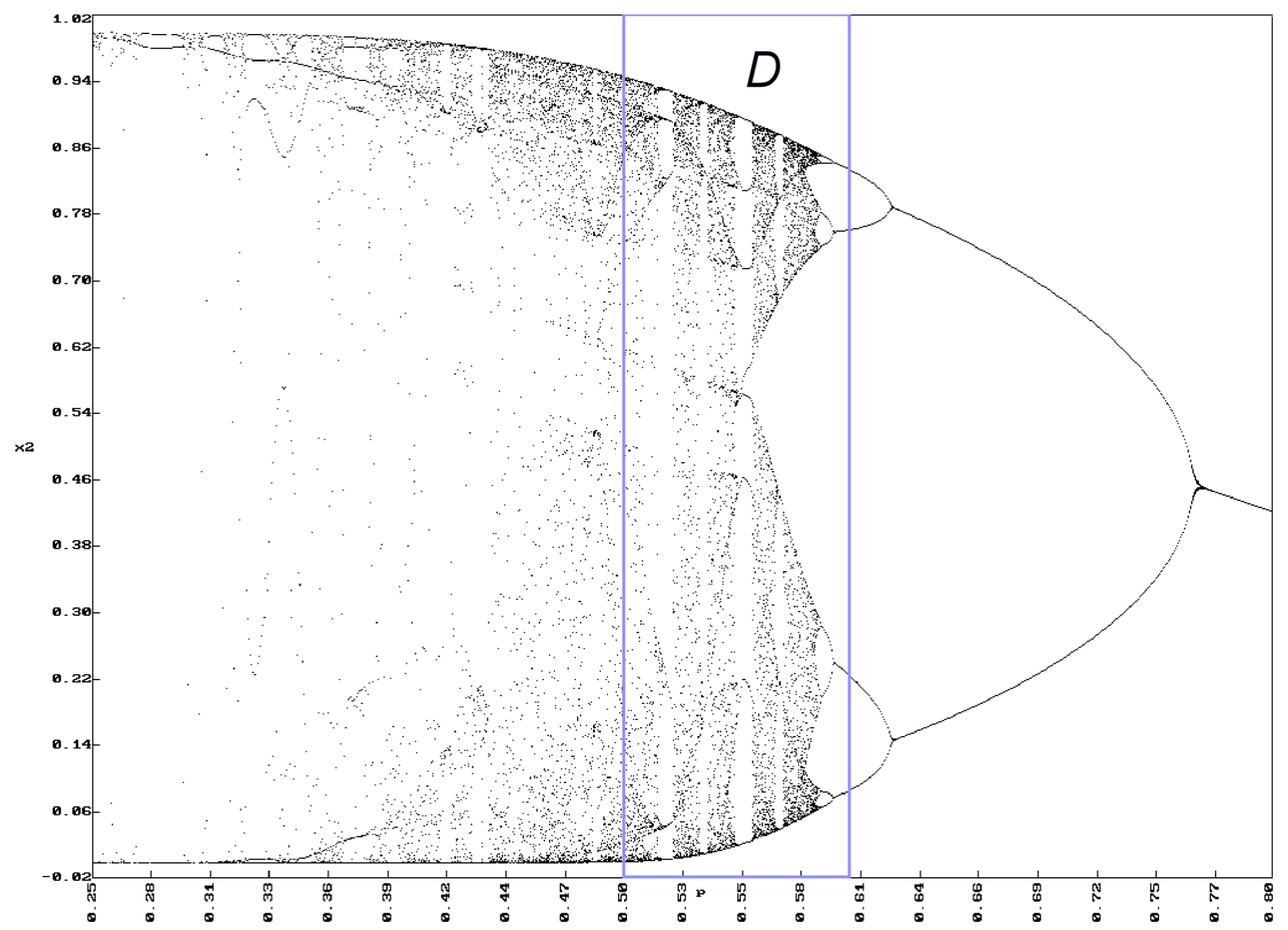

Fig. 1. Bifurcation diagram for $x_{2}$.

approximation of any attractor (chaotic or not) of a dynamical system belonging to a class of systems depending linearly on the control parameter $p$. For this purpose, $p$ is switched within the set $P_{N}$ for some finite short period of time while the system is numerically integrated on some interval of time $I=[0, T], T>0$. The obtained attractor is a numerical approximation of one the systems's attractors.

The class of systems, where the algorithm has been applied, is modeled by the following autonomous initial value problem

$$
\dot{x}=f(x)+p A x, \quad x(0)=x_{0}, t \in I,
$$

where $f: \mathbf{R}^{n} \rightarrow \mathbf{R}^{n}$ is a function continuous with respect to the state variable, $x \in \mathbf{R}^{n}$ and $A=\left(a_{i, j}\right)_{n \asymp n}$ is a real constant matrix.

To implement numerically the PS algorithm, the length of the time intervals in which $p$ is switched may be taken as multiple of $h: m_{i} h$ for $m_{i}, i=1,2, \ldots, N$, some positive integers [Danca et al., 2011] (see the pseudo code in Table 1)

Under the uniqueness assumption on IVP (2), to each $p$ and initial conditions there corresponds a unique attractor (which can be chaotic or regular (here stable limit cycles of fixed points)). Now it is easy to understand why PS algorithm is a generalization of Parrondo's paradox according to which losing strategies can win i.e. it is possible to mix two losing games into a winning combination: "losing + losing $=$ winning" [Harmer et al., 2000] or, in other words: "two ugly parents can have beautiful children" (Doron Zeilberger on receiving the 1998 Leroy P. Steele Prize). If by "loosing" gain one consider "chaos" and by "winning" gain, "regular", we obtain the following form: "chaos+chaos=regular" which is actually 


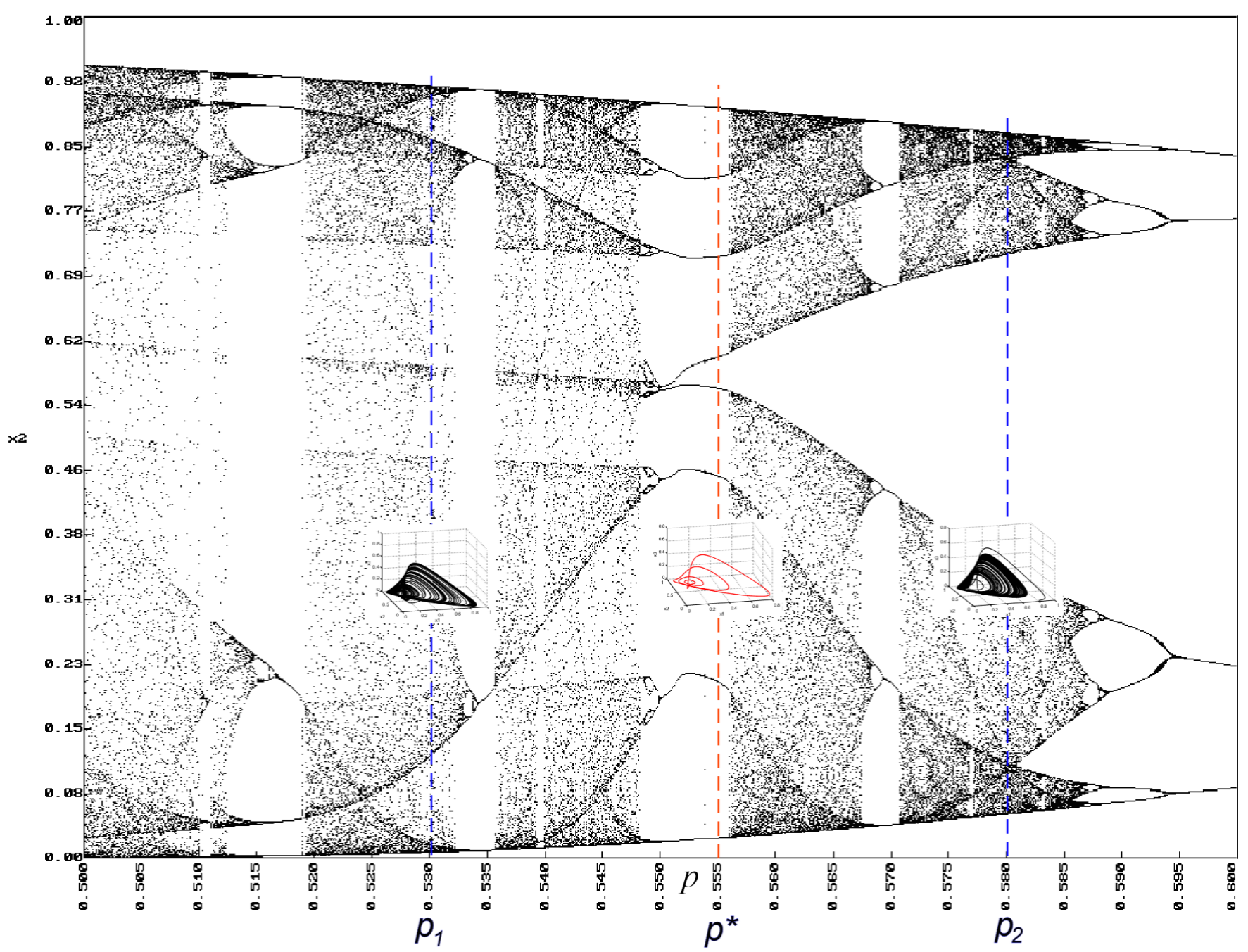

Fig. 2. Detail $D$ of bifurcation diagram.

the simplest variant for PS algorithm. Thus, if $p$ is alternated within the set $\left\{p_{1}, p_{2}\right\}$ with $p_{1}$ and $p_{2}$ corresponding to chaotic attractors, it is possible to obtain a regular attractor but also a chaotic one. Obviously, there are several other possibilities such as: "regular+regular $=$ chaos", "regular+chaos=regular" and so on. Therefore, switching $p$ with PS algorithm while the IVP is integrated, within whatever set of values, actually we alternate between regular and chaotic attractors, i.e. a generalization of Parrondo's paradox, since the result can be a regular attractor or a chaotic one.

The initial value problem (2) is enough general to include, beside the system modeled by (1), the great generality of known systems such as Lorenz, Chen, Rössler, Lü, some classes of networks and so on but also systems modeling the course of some epidemics (such as SIR models [Kermack \& McKendrick, 1927]). Moreover, on our knowledge, stabilizing the behavior of a model describing cancer behavior, by switching the control parameter represents a novel approach.

In this letter the attractors will be considered simply as trajectories after neglecting a sufficiently long period of transients [Foias et al., 1995].

As shown in [Danca et al., 2011], the attractor obtained with PS algorithm, denoted $A^{*}$, is approximatively identical to the attractor, denoted $A_{p^{*}}$, obtained by integrating the initial value problem for $p=p^{*}$ 


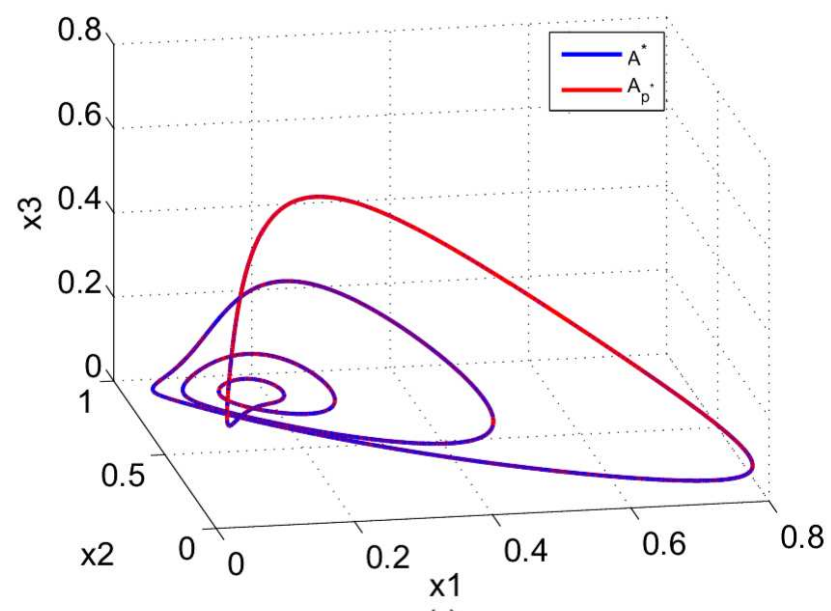

(a)

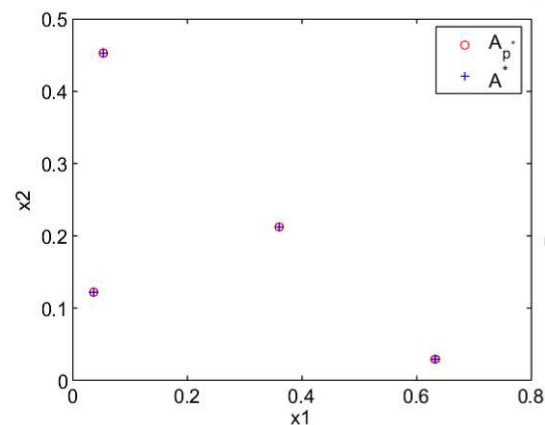

(b)

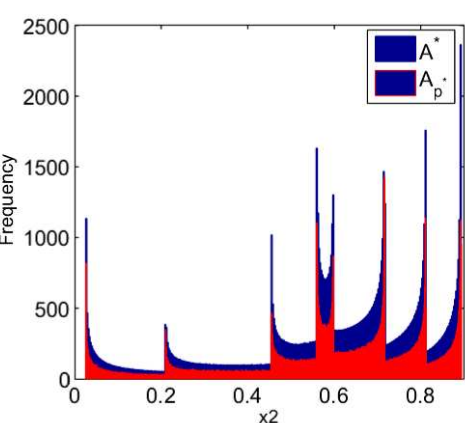

(c)

Fig. 3. Stable limit cycle obtained with AS algorithm for $p_{1}=0.53$ and $p_{2}=0.58$ for which $p^{*}=0.555$. $A^{*}$ is plotted in red while $A_{p^{*}}$ in blue: (a) Overlapped phase portraits. (b) Overlapped Poincaré sections with the plane $x_{3}=0.3$. (c) Overlapped histograms.

with $p^{*}$ given by the following expression

$$
p^{*}=\frac{\sum_{i=1}^{N} m_{i} p_{i}}{\sum_{i=1}^{N} m_{i}} .
$$

The algorithm is useful when, for objective reasons, some values for $p$ cannot be set and we want to obtain the underlying attractor, avoiding the tedious calculations implied by the known chaos control and anticontrol algorithms. Also, the algorithm may be used to explain some natural processes in Nature when, accidently, the parameters may switch within a set of values.

In this letter we give computationally evidence of our results, namely we show that in phase portraits,

Table 1. Pseudo-code of the $P S$ algorithm.

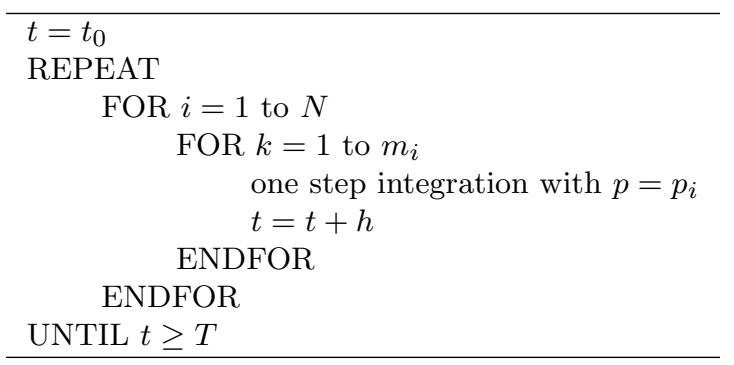


time series, histograms and Poincaré sections, the trajectories of $A^{*}$ and $A_{p^{*}}$ are approximatively identical and Hausdorff distance between trajectories is of order of $10^{-3 \div-5}$ (for the analytical proof see [Danca et al., 2011]).

\section{Finding stable attractors}

Because stable motions in the case of systems like those modeled by (1) are essential, in this letter we are interested in finding the stable regular behaviors.

To obtain a stable trajectory of (1), we have to chose (helped e.g. by bifurcation diagram) a desired value $p^{*}$ for which the system behaves regularly and, for fixed $h, N$ and $P_{N}$, find the values $m_{i}, i=1,2, \ldots, N$, such that $p^{*}, m_{i}$, and $p_{i}$ verify (3). Next, the PS algorithm is applied. ${ }^{1}$

Let chose for example $p^{*}=0.555$ for which the system behaves on a stable limit cycle (Figure 2) and $P_{2}=\{0.53,0.58\}$ according to which the system behaves chaotically. A solution to (3) is, for example, $m_{1}=m_{2}=1$. Thus, $p^{*}=0.555=\left(1 \times p_{1}+1 \times p_{2}\right) / 2=(0.53+0.58) / 2$. The identity between $A^{*}$ and $A_{p^{*}}$ can be seen from the overlapped phase portraits, histograms, and Poincaré sections (Figures 3 (a), (b) and (c) respectively). The Hausdorff distance (calculated as in [Falconer, 1990, p 114]) is $D_{H}=2 \times 10^{-5}$ which confers a good approximation ${ }^{2}$. Therefore, a characteristic stable motion was obtained by switching $p$ between $p_{1}$ and $p_{2}$, which in Parrondo's paradox terms means: "chaos+chaos=regular".

Due to the non-unicity in (3), the same attractor can be obtained with other switching schemes. For example, the same stable limit cycle corresponding to $p^{*}=0.555$ can be obtained with $P_{5}=$ $\{0.198,0.302,0.402,0.710,0.806\}$ (for which we have, respectively, "regular", "regular", "chaos", "regular", "regular"), and $m_{1}=m_{2}=2, m_{3}=1, m_{4}=4$ and $m_{5}=3$ since equation (3) is again verified (Figure 4). If we denote "chaos" by $\mathrm{C}$ and "regular" by $\mathrm{R}$, we have: " $2 \mathrm{R}+2 \mathrm{R}+\mathrm{C}+4 \mathrm{R}+3 \mathrm{R}=\mathrm{R}$ ". However, this time, even the two attractors, $A^{*}$ and $A_{p^{*}}$ are well matched, one can be seen some difference between them (see the detail in Figure 4) and the trajectory of $A^{*}$ spirals around that of $A_{p^{*}}$.

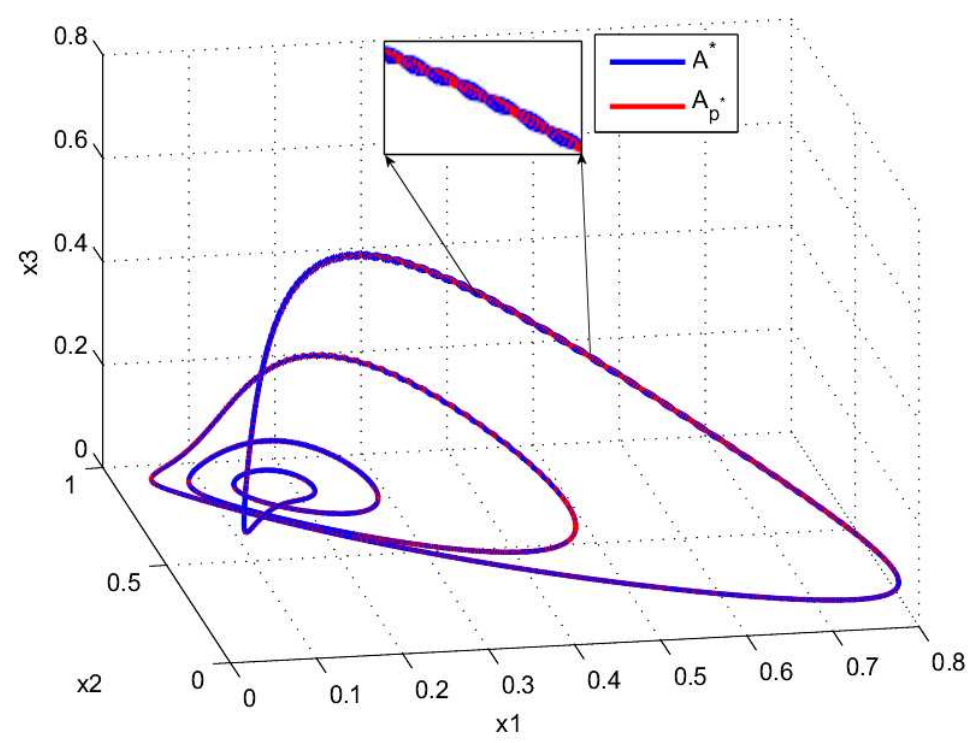

Fig. 4. Phase portrait of the same stable limit cycle for $p^{*}=0.555$ obtained by switching the parameter values $0.198,0.302,0.402,0.710,0.806$. The detail shows that $A^{*}$ spirals around $A_{p^{*}}$.

\footnotetext{
${ }^{1}$ Obviously, (3) has several solutions.

${ }^{2}$ This means that $A^{*}$ belongs inside a three-dimensional $\varepsilon-$ tube around $A_{p}^{*}$, with $\varepsilon=2 \times 10^{-5}$.
} 

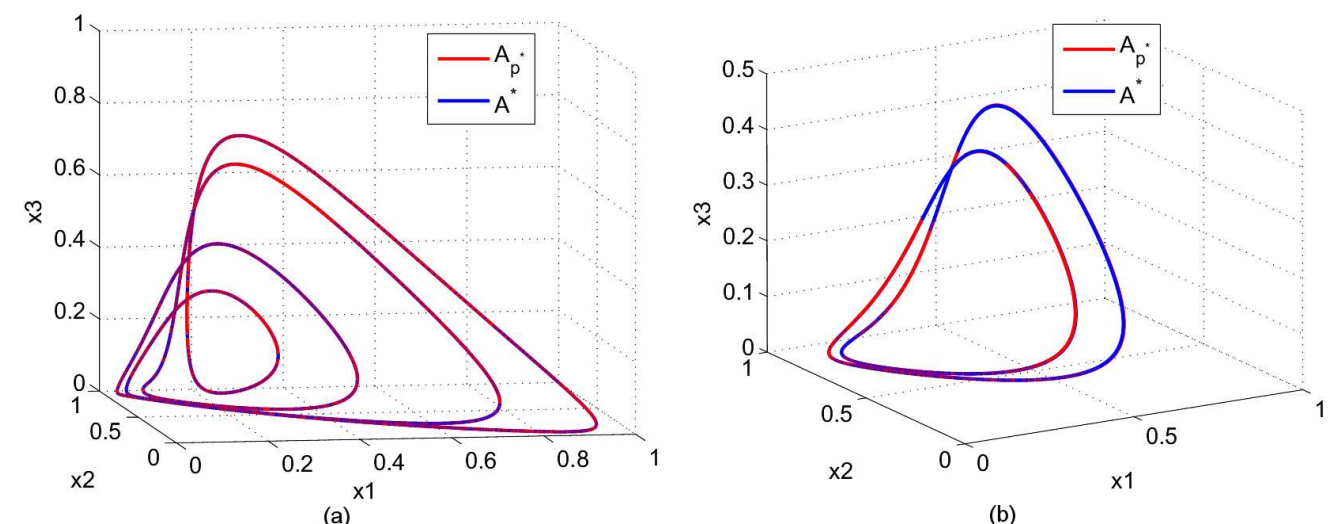

Fig. 5. Two synthesized stable limit cycles corresponding to $p^{*}=0.517$ (a) and $p^{*}=0.615$ (b).

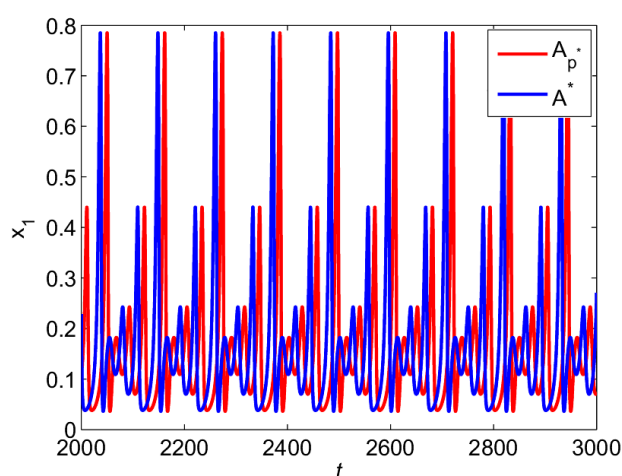

(a)

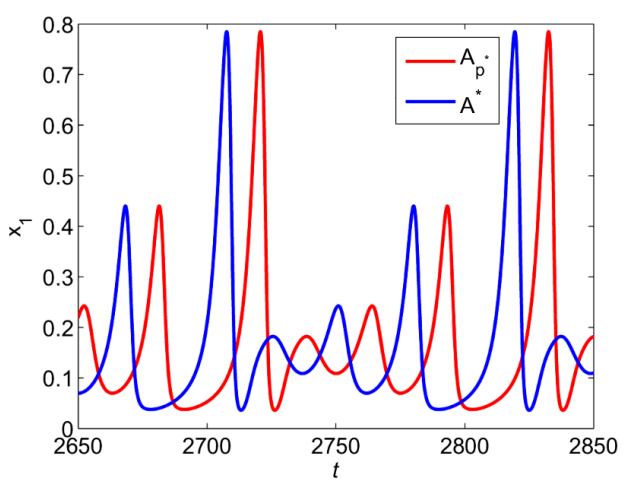

(b)

Fig. 6. Time series of $x_{1}$ for the trajectories corresponding to $A^{*}$ and $A_{p^{*}}$ for $p^{*}=0.555$. (a) The two trajectories are shifted in time one to each other. (b) Detail.

Other two stable limit cycles obtained with PS algorithm, corresponding to $p^{*}=0.517$ and $p^{*}=0.615$ with $P_{2}^{\prime}=\{0.505,0.523\}, m_{1}=m_{2}=1$ and $P_{2}^{\prime \prime}=\{0.45,0.78\}, m_{1}=m_{2}=1$ respectively, are presented in Figure 5 (a) and (b) respectively.

As can be seen in Figure 6 for the time series of the case presented in Figure 3, the trajectories corresponding to $A^{*}$ and $A_{p^{*}}$ are time shifted one to each other. We have remarked that this shift takes place in all studied examples and can be forward or backward, function on $m_{i}$ and the order position of $p^{*}$ within the set $P_{N}$ [Danca et al., 2011].

For a fixed set $P_{N}$, due to the convexity of (3), even random choices for $m_{i}$ may leads to the same result: $A^{*}$ is an attractor belonging to the set of all attractors for the considered system (obvious, the values should be chosen with some care due to the inherent numerical errors). For example, while PS runs, choosing randomly (with uniform distribution) $m_{i}$ within $\{0,1\}$, by switching the values $0.505+k * 0.01$ for $k=0,1, \ldots, 10$, with $k \neq 5$, again $A^{*}$ and $A_{p^{*}}$ match (Figure 7). However, as expected, the difference between the two trajectories is bigger now and the Hausdorff distance is $D_{H}=4.6 \times 10^{-4}$ (see detail in Figure 7).

Using the same way, chaotic attractors can be synthesized too. For example, a chaotic behavior corresponds to $p^{*}=0.544$ and can be obtained switching $p$ within the values $p_{1}=0.535$ and $p_{2}=0.553$ with $m_{1}=m_{2}=1$ (Figure 8). Now, because $A^{*}$ is chaotic, the underlying solution is obtained, theoretically, by infinite period of time. Therefore, as can be seen in Figure 8, there are some relative small differences between the two attractors, even the two attractors still match. Hausdorff distance calculated for the first 300000 points, as expected, is bigger: $d_{H}=2 \times 10^{-3}$. 


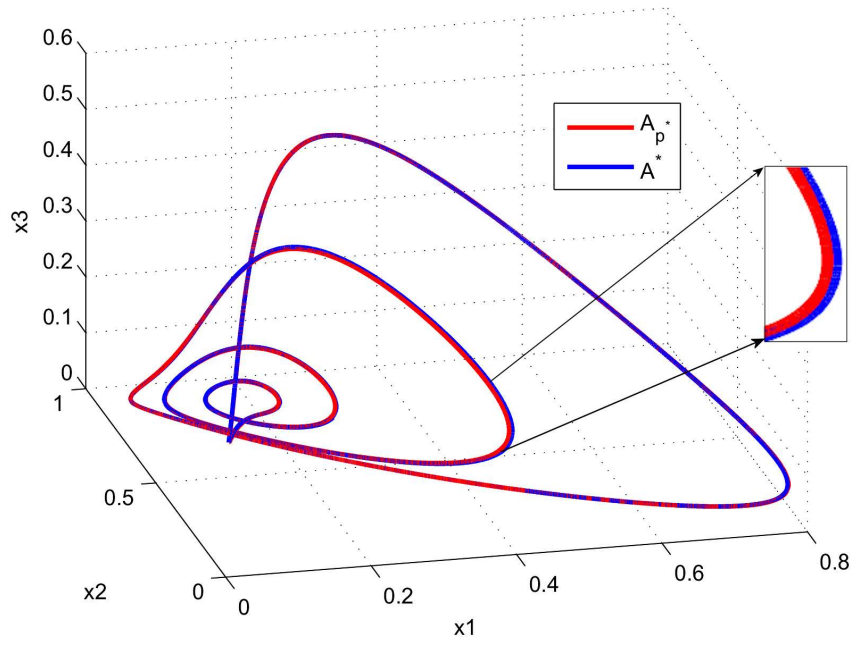

Fig. 7. Random synthesis of $A^{*}$ for $p^{*}=0.555$ with ten values for $p$.

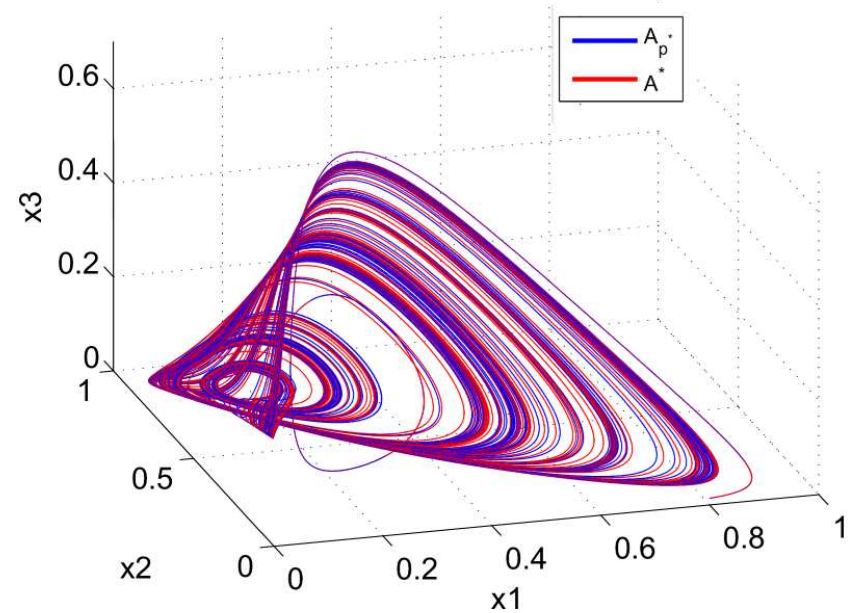

(a)

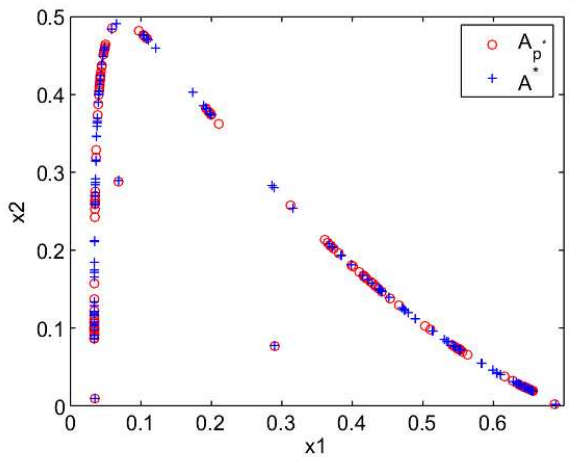

(b)

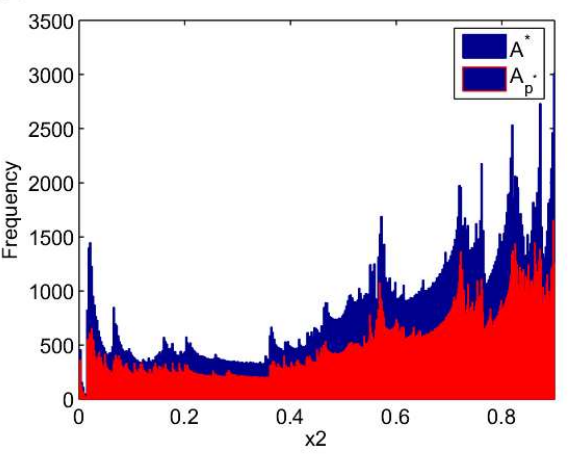

(c)

Fig. 8. $\quad A^{*}$ and $A_{p^{*}}$ for the chaotic attractor obtained by switching the values $p_{1}=0.535$ and $p_{2}=0.553$. (a) Overlapped phase portraits. (b) Overlapped Poincaré sections with the plane $x_{3}=0.3$. (c) Overlapped histograms. 


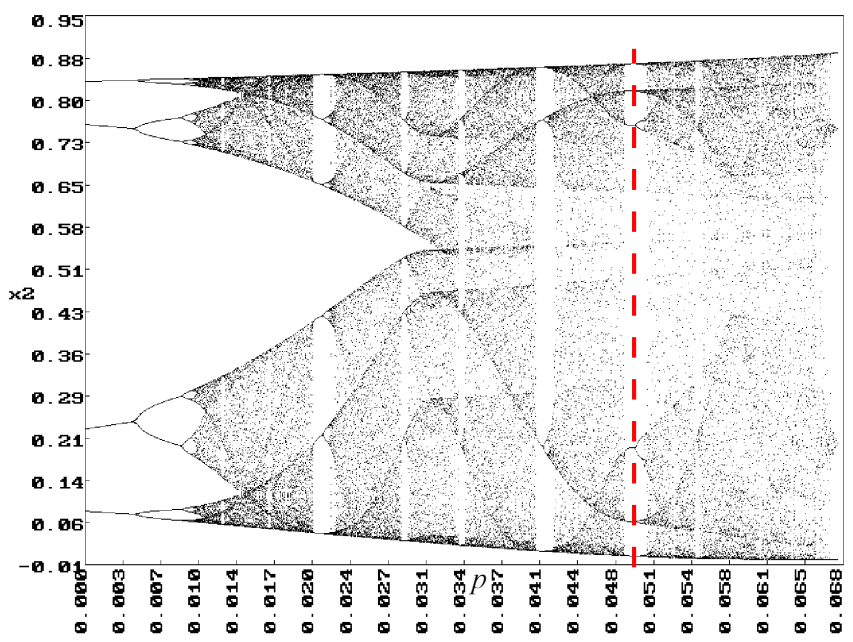

(a)

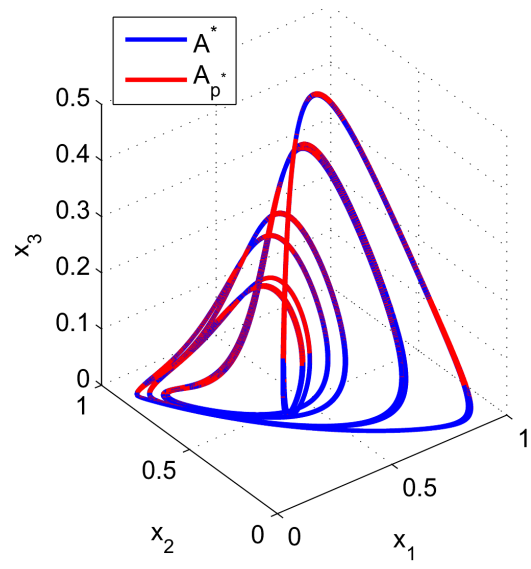

(b)

Fig. 9. (a) Bifurcation diagram for modified system 4; (b) Stable multiple cycle corresponding to $p^{*}=0.05$ and scheme $[1 p 1,1 p 2,1 p 3,1 p 4,1 p 5,1 p 6,4 p 7,4 p 8]$ for $P_{N}=\{0.015,0.024,0.030,0.037,0.045,0.053,0.060,0.064\}$

The PS algorithm can also be applied to more other cancer growth models such as the following generalization of (1) under drug therapy, with the first equation modified (perturbed) as follows

$$
\dot{x}_{1}=x_{1}\left(1-x_{1}\right)-x_{1} x_{2}-2.5 x_{1} x_{3}-p x_{1},
$$

where $p x_{1}$ is a control (perturbation) of the tumor growth with log-kill effect via drug therapy (see [Pillis \& Radunskaya, 2003] or [Itik et al., 2009]). Now the PS algorithm can be applied to control the cancer growth through the drug therapy. As can be seen in Figure 9 a, with this modification, the bifurcations does not reverse direction as for the system (1).

For example if we take $P_{N}=\{0.015,0.024,0.030,0.037,0.045,0.053,0.060,0.064\}$ and the scheme $\left[1 p_{1}, 1 p_{2}, 1 p_{3}, 1 p_{4}, 1 p_{5}, 1 p_{6}, 4 p_{7}, 4 p_{8}\right]$ one can obtain the stable behavior corresponding to $p^{*}=0.05$ (Figure $9 \mathrm{~b})$.

\section{References}

Danca M.-F., Romera M., Pastor G. \& Montoya F. [2011] "Finding Attractors of Continuous-Time Systems by Parameter Switching," Nonlinear Dynamics, accepted.

Falconer K. [1990] Fractal Geometry, Mathematical Foundations and Applications (John Wiley \& Sons, Chichester).

Foias C. \& Jolly M. S. [1995] "On the numerical algebraic approximation of global attractors," Nonlinearity 8, 295-319.

Gatenby R.A. \& Maini P.K. [2003] "Mathematical oncology: Cancer summed up," Nature 421 (6921), 321.

Harmer G. P., Parrondo J. M. R. \& Abbott D. [2000] "New paradoxical games based on brownian ratchets," Physical Review Letters 85, 5226-5229.

Kermack W. O. \& McKendrick A. G. [1927] "A Contribution to the Mathematical Theory of Epidemics." Proc. Roy. Soc. Lond. A 115, 700-721.

Itik M. \& Banks S. P. [2010] "Chaos in a three-dimenisioonal cancer model," Int. J. Bifurcation and Chaos 20 (1), 71-79.

Itik M., Salamci M. U. \& Banks S. P. [2009] "Optimal control of drug therapy in cancer treatment," Nonlinear Analysis 71 e1473-e1486.

De Pillis L. G. \& Radunskaya A. [2003] "The dynamics of an optimally controlled tumor model: A case study," Mathematical and Computer Modelling 37 (11), 1221-1244.

Roose T., Chapman S.J. \& Maini P.K. [2007] "Mathematical Models of Avascular Tumor Growth," SIAM Rev. 49(2), 179-208. 